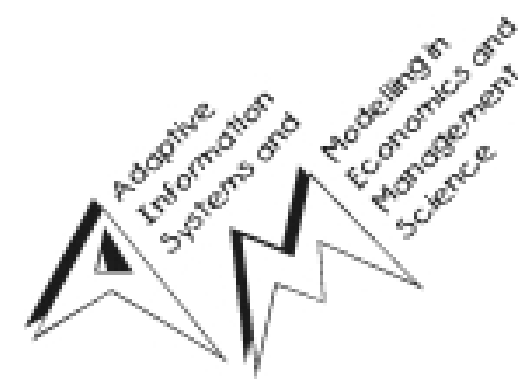

June 2001

SFB

'Adaptive Information Systems and Modelling in Economics and Management Science'

Vienna University of Economics and Business Administration

Augasse 2-6, 1090 Wien, Austria

in cooperation with

University of Vienna

Vienna University of Technology

http://www.wu-wien.ac.at/am

Papers published in this report series are preliminary versions of journal articles and not for quotations.

This piece of research was supported by the Austrian Science Foundation (FWF) under grant SFB\#010 ('Adaptive Information Systems and Modelling in Economics and Management Science'). 


\title{
Nonlinear Adaptive Beliefs and the Dynamics of Financial Markets: The Role of the Evolutionary Fitness Measure
}

\author{
Andrea Gaunersdorfer ${ }^{1}$ and Cars H. Hommes ${ }^{2}$ \\ 1 Department of Business Studies, University of Vienna \\ Brünner Straße 72, A-1210 Wien, Austria \\ Andrea.Gaunersdorfer@univie.ac at \\ WWW home page: http://finance2.bwl .univie.ac at \\ 2 CeNDEF, University of Amsterdam \\ Roetersstraat 11, NL-1018 WB Amsterdam, The Netherlands \\ hommes@fee.uva.nl \\ WWW home page: http://www.fee.uva.nl/cendef/
}

\begin{abstract}
We introduce a simple asset pricing model with two types of adaptively learning traders, fundamentalists and technical traders. Traders update their beliefs according to past performance and to market conditions. The model generates endogenous price fluctuations and captures some stylized facts observed in real returns data, such as excess volatility, fat tails of returns distributions, volatility clustering, and long memory. We show that the results are quite robust w.r.t. to different choices for the performance measure.
\end{abstract}

\section{Introduction}

Traditional models in finance rest on the assumptions of informationally efficient markets (efficient market hypothesis, EMH) and rational agents that have symmetric information, identical (homogenous) preferences and build their expectation on all available information (rational expectations hypothesis). In a world with rational expectations and efficient markets everything is driven by unanticipated uncorrelated shocks (technological shocks, changing preferences, etc.). However, these models fail to explain many so-called stylized facts observed in financial time series, for example, excess volatility, fat tailed distributions of stock returns, and volatility clustering (that is, high/small price changes are mostly followed by high/small price changes). Though asset returns contain little serial correlation (which is consistent with the weak form of the EMH) there is substantially more correlation between absolute or squared returns. These facts indicate that prices in financial markets are not solely determined by economic fundamentals. Markets seem to have internal dynamics of their own and are influenced by market psychology and investors 'animal spirits'.

In the last decade an increasing number of models with heterogeneous, boundedly rational agents has been introduced. Such models typically distinguish be- 
tween two types of traders, fundamentalists, who believe that prices are determined by economic fundamentals, and chartists, who believe that prices can be predicted by simple trading rules based upon past price patterns in the recent past.

Brock and Hommes [BH1] have introduced the concept of Adaptive Belief Systems. Agents adapt their predictions by choosing among a finite number of predictors (beliefs, expectations), which are functions of past information. Based on a performance measure attached to every predictor, which is publically available, agents make a boundedly rational choice between the predictors. This leads to the so-called Adaptive Rational Equilibrium Dynamics, a nonlinear, evolutionary dynamics across competing beliefs or trading strategies, coupled to the dynamics of the endogenous variables. In a series of papers Brock and Hommes [BH2], [BH3], [BH4], Gaunersdorfer [G1], and Gaunersdorfer and Hommes [GH] have applied this concept to a simple asset pricing model. For a comprehensive survey on these models see $[\mathrm{H}]$.

In this paper we review the model of $[\mathrm{GH}]$ and focus on the influence of different performance measures on the dynamics. In section 2 we recall the model and discuss a second evolutionary fitness measure. Section 3 concludes.

\section{The Model}

Our adaptive belief system is based on a standard asset pricing model extended to heterogeneous beliefs. Agents can either invest in a riskless asset, which is perfectly elastically supplied with gross rate of return $R$, or in a risky asset with price $p_{t}$ per share (ex-dividend), which pays a stochastic dividend $y_{t}$ at time $t$. Agents are assumed to be mean-variance maximizers, so that the demand $z_{h t}$ of trader type $h$ for the risky asset solves

$$
\max _{z_{h t}}\left\{E_{h t}\left(W_{h, t+1}\right)-\frac{a}{2} V_{h t}\left(W_{h, t+1}\right)\right\}
$$

where $W_{h, t+1}=R W_{h t}+\left(p_{t+1}+y_{t+1}-R p_{t}\right) z_{h t}=: R W_{h t}+R_{t+1} z_{h t}$ describes wealth dynamics and $E_{h t}$ and $V_{h t}$ denote the beliefs or forecasts of trader type $h$ about conditional expectation $E_{t}$ and conditional variance $V_{t}$, based on a publically available information set such as past prices and dividends. $a>0$ is the risk aversion parameter, $R_{t}=p_{t}+y_{t}-R p_{t-1}$ is the excess return per share. Assuming that beliefs about conditional variances are constant, ${ }^{1} V_{h t} \equiv \sigma^{2}$, demand for the risky asset is given by

$$
z_{h t}=\frac{E_{h t} R_{t+1}}{a \sigma^{2}}
$$

Let $z_{s t}$ and $n_{h t}$ denote supply of outstanding shares of the risky asset per investor and fraction of trader type $h$, respectively. Equilibrium of demand and supply

\footnotetext{
${ }^{1}$ [G1] studies a model version with time varying beliefs about conditional variances and shows that the dynamics are quite similar as in the case with constant beliefs.
} 
yields

$$
\sum_{h} n_{h t} z_{h t}=z_{s t}
$$

We focus on the special case of zero suppy of outside shares, $z_{s t} \equiv 0$. Further assume that dividends follow an iid process with constant mean $E_{t}\left(y_{t+1}\right) \equiv$ $E\left(y_{t}\right) \equiv \bar{y}$ and traders have homogeneous beliefs about future dividends $E_{h t}\left(y_{t+1}\right)$ $=E_{t}\left(y_{t+1}\right)=\bar{y}$. Thus, the market equilibrium equation (3) can be rewritten as

$$
R p_{t}=\sum_{h} n_{h t} E_{h t}\left(p_{t+1}\right)+\bar{y} .
$$

Market equilibrium thus states that the price of the risky asset equals the discounted sum of tomorrow's expected price and tomorrow's expected dividends, averaged over all trader types.

Let us first consider the special case where traders have homogeneous rational expectations, that is, $E_{h t}\left(p_{t+1}\right)=E_{t}\left(p_{t+1}\right) \forall h$. This allows us to define a benchmark 'fundamental solution' (EMH fundamental rational expectations price)

$$
p^{*}=\sum_{k=1}^{\infty} \frac{\bar{y}}{R^{k}}=\frac{\bar{y}}{R-1} .
$$

Note that in case of homogeneous rational expectations (5) is the only solution of (4) which fulfills the transversality condition $\lim _{t \rightarrow \infty} E_{t}\left(p_{t+k}\right) / R^{k}=0$ ('no bubbles condition').

We consider a simple example with two types of traders choosing simple linear prediction rules. The first type are so-called fundamentalists, believing that prices will move towards the fundamental rational expectations value $p^{*}$,

$$
E_{1 t}\left(p_{t+1}\right)=p_{1, t+1}^{e}=p^{*}+v\left(p_{t-1}-p^{*}\right), \quad 0 \leq v \leq 1 .
$$

In the special case $v=1$, traders use the latest observed price as predictor, $E_{1 t}\left(p_{t+1}\right)=p_{1, t+1}^{e}=p_{t-1}$. We call this type of traders EMH believers, since the naive forecast is consistent with an efficient market, where prices follow a random walk. The second type of traders are technical traders extrapolating the latest observed price change,

$$
E_{2 t}\left(p_{t+1}\right)=p_{2, t+1}^{e}=p_{t-1}+g\left(p_{t-1}-p_{t-2}\right), \quad g \geq 0 .
$$

Market equilibrium equation (4) now becomes

$$
R p_{t}=n_{1 t}\left(p^{*}+v\left(p_{t-1}-p^{*}\right)\right)+n_{2 t}\left(p_{t-1}+g\left(p_{t-1}-p_{t-2}\right)\right)+\bar{y}+\varepsilon_{t},
$$

where a dynamic iid noise term $\varepsilon_{t}$ has been added to equation (4), representing model approximation error or noise coming from noise traders, traders whose behavior is not explained by the model but considered as exogenously given. 
The second, conditionally evolutionary part of the model describes how beliefs, i.e. fractions, change over time. The basic idea is that fractions are updated based upon a performance measure of forecasting rules, conditioned on the deviation of the actual price from the fundamental price $p^{*}$. In the first step (evolutionary part) fractions are determined as discrete choice probabilities according to past performance or fitness,

$$
\tilde{n}_{h t}=\exp \left(\beta U_{h, t-1}\right) / Z_{t-1}, \quad Z_{t-1}=\sum_{h} \exp \left(\beta U_{h, t-1}\right),
$$

where $Z_{t-1}$ is a normalization factor such that fractions add up to one. $U_{h t}$ ist the 'fitness function' or 'performance measure'. $\beta \geq 0$ is called 'intensity of choice', it measures how fast the mass of traders will switch to the optimal strategy. [BH1], [BH2], and [G1] show that this parameter plays a crucial role in the route to complicated dynamical behavior.

A natural candidate for evolutionary fitness is (accumulated) realized (excess) profits $R_{t} z_{h t}$, given by

$$
U_{h t}^{(1)}=\frac{1}{a \sigma^{2}}\left(p_{t}+y_{t}-R p_{t-1}\right)\left(p_{h t}^{e}+\bar{y}-R p_{t-1}\right)+\eta U_{h, t-1},
$$

where $0 \leq \eta \leq 1$ is a memory parameter measuring how fast past realized fitness is discounted for strategy selection and $z_{h t}$ is given by (2).

However, this performance measure is inconsistent with investors being risk averse mean-variance maximizers, since it ignores the variance term in (1). Thus, another natural candidate for the fitness function is utility derived from realized profits, that is, risk adjusted realized profits. Utilities of realized profits in period $t$ of investortype $h$ are given by

$$
\pi_{h t}:=\pi\left(R_{t+1}, E_{h t}\left[R_{t+1}\right]\right)=R_{t+1} z\left(E_{h t}\left[R_{t+1}\right]\right)-\frac{a}{2} \sigma^{2} z^{2}\left(E_{h t}\left[R_{t+1}\right]\right)
$$

where

$$
z\left(E_{h t}\left[R_{t+1}\right]\right)=\arg \max \pi_{h t}=\frac{E_{h t}\left[R_{t+1}\right]}{a \sigma^{2}}=z_{h t} .
$$

Note that discrete choice probabilities are independent of utility level, i.e. they do not change if the same term $\pi_{t}$ is subtracted off all exponents in (9). Thus, we may consider

$$
\begin{aligned}
\pi_{h t}-\pi_{t} & =\pi\left(R_{t+1}, E_{h t}\left[R_{t+1}\right]\right)-\pi\left(R_{t+1}, R_{t+1}\right) \\
& =-\frac{1}{2 a \sigma^{2}}\left(E_{h t}\left[R_{t+1}\right]-R_{t+1}\right)^{2} \\
& =-\frac{1}{2 a \sigma^{2}}\left(p_{t+1}-p_{h, t+1}^{e}+\delta_{t+1}\right)^{2}
\end{aligned}
$$

where $\pi_{t}=\pi\left(R_{t+1}, R_{t+1}\right)$ is the utility of profits of perfect foresight investors and $\delta_{t}$ is the noise term of the stochastic dividend process, i.e. $y_{t}=\bar{y}+\delta_{t}$. Note that in the absence of random shocks, $\delta_{t} \equiv 0$, these differences simply reduce 
to a negative constant times the squared prediction errors. Thus, we define the second fitness measure as

$$
U_{h t}^{(2)}=-\frac{1}{2 a \sigma^{2}}\left(p_{t}-p_{h t}^{e}+\delta_{t+1}\right)^{2}+\eta U_{h, t-1} .
$$

In the second step of updating of fractions, technical traders condition their charts upon information about fundamentals, that is, on price deviations from the fundamental value $p^{*}$,

$$
n_{2 t}=\tilde{n}_{2 t} \exp \left[-\left(p_{t-1}-p^{*}\right)^{2} / \alpha\right], \quad \alpha>0, \quad n_{1 t}=1-n_{2 t} .
$$

As long as prices are close to the fundamental value, fractions are almost completely determined by evolutionary fitness. However, when price deviations from the fundamental become large the correction term $\exp \left[-\left(p_{t-1}-p^{*}\right)^{2} / \alpha\right]$ becomes small, representing the fact that more and more traders believe that a correction towards the fundamental is about to occur.

The noisy conditional evolutionary asset pricing model with fundamentalists versus technical traders is now given by (8), (9), (12) and one of the evolutionary performance measures (10) or (11). This defines a six-deminsional (in the special case of $\eta=0$ a four-dimensional) dynamical system. A detailed bifurcation analysis of the deterministic skeleton (where $\varepsilon_{t} \equiv 0$ and $\delta_{t} \equiv 0$ ) for performance measure (11) is given in [GHW]. Figure 1 gives an overview of the dynamics (our simulations show that the model with performance measure (10) has similar properties, in particular, local behavior around the fundamental steady state is the same). The fundamental value $p^{*}$ is a unique steady state which is stable for $g<2 R$. As $g$ is increased it is destabilized by a Hopf bifurcation at $g=2 R$ and a stable invariant circle with periodic or quasi-peridic dynamics emerges. This circle may undergo bifurcations as well, turning into a strange attractor.

For some value of $v=: v_{\mathrm{Ch} 1}$ on the Hopf bifurcation line $g=2 R$ the Hopf bifurcation changes from super- to subcritical, a so-called Chenciner bifurcation occurs. That is, when the Hopf bifurcation line is crossed from right to left (decreasing $g$ beyond $2 R$ ) for $v_{\mathrm{Ch} 1}<v<v_{\mathrm{Ch} 2}$ an unstable invariant circle emerges out of the (unstable) equilibrium and the equilibrium becomes stable, wheras the stable invariant circle created in the supercritical Hopf bifurcation still exists. Decreasing $g$ further, the stable und unstable circles collide and disappear in a saddle-node bifurcation of invariant circles at curve $S$. Thus, there is a parameter region where a stable equilibrium and a stable invariant circle coexist, bounded by the Hopf bifurcation line and curve $S$. When the system is buffeted with dynamic noise irregular switching between close to fundamental steady state fluctuations (with small volatility) and (almost) periodic price fluctuations (with high volatility) occurs. We call this region 'volatility clustering region'.

A second phenomenon in our model is intermittency, that is, small (almost regularly) price fluctuations are suddenly interrupted by large price fluctuations triggered by technical trading. On the right hand side of figure 1 a strange attractor and the corresponding price series are plotted, showing intermittent chaos. Both phenomena, coexistence of attractors and intermittency, appear to be extremly well suited as description of the phenomenon of volatility clustering. 


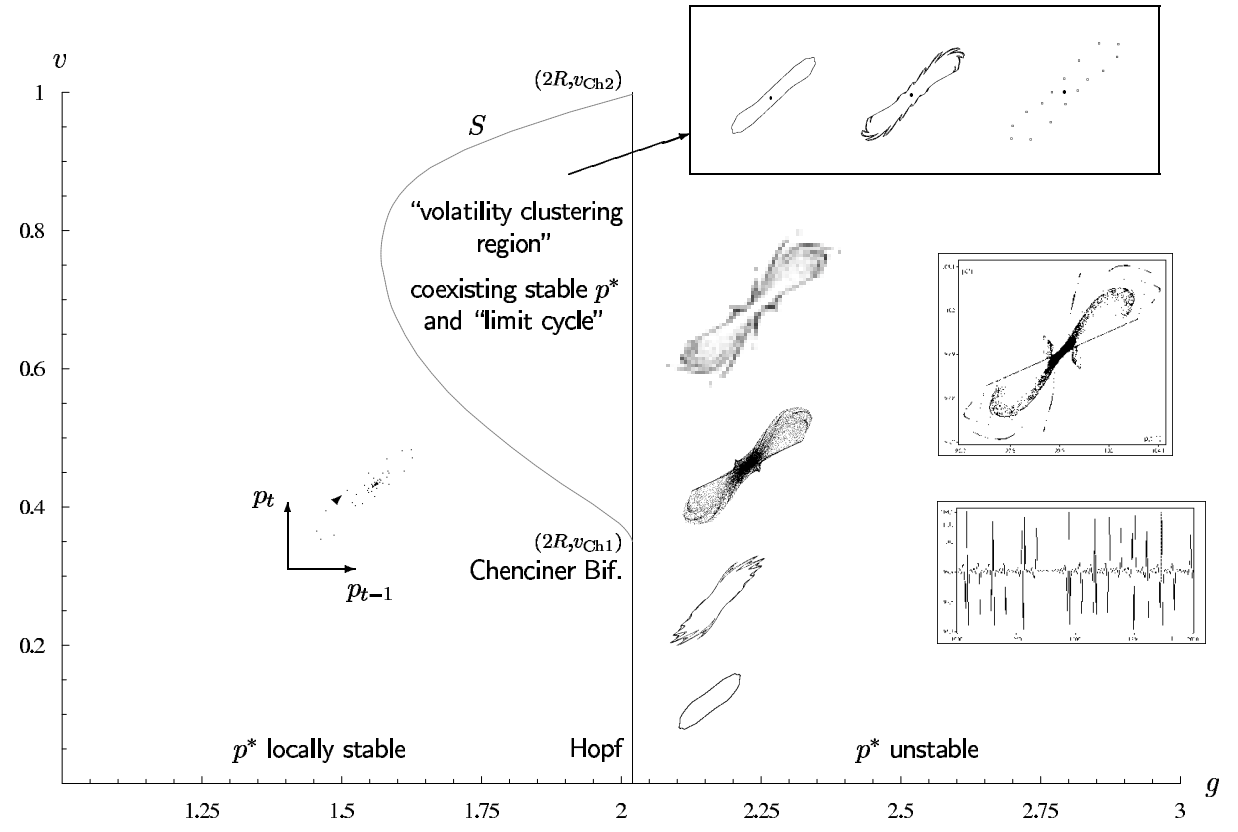

Fig. 1. Bifurcation diagram in the $g$ - $v$-plane. Projections of attractors to the $p_{t-1}-p_{t^{-}}$ plane in the corresponding parameter regions are plotted. At points $\left(2 R, v_{\mathrm{Ch} i}\right), i=1,2$, Chenciner bifurcations occur. These points lie on the Hopf bifurcation line $g=2 R$ and are connected by a curve $S$ corresponding to a saddle-node bifurcation curve of invariant circles. Parameter values: $\beta=3, R=1.01, \bar{y}=1, \alpha=10, a \sigma^{2}=1$, parameter values for the attractor and corresponding price series on the right hand side, showing intermittent chaos: $\beta=20, g=2.1, v=0.5$.

Figure 2 compares returns series of daily S\&P 500 data over 40 years with those generated by our model. In the chosen examples parameter $v$ is set equal to 1, that is, EMH-believers and technical traders interact. In these examples prices are highly persistent and the system is close to having an unit root. In fact, in case $v=R=1$ the characteristic polynomial of the Jacobian at the steady state $p^{*}$ has an eigenvalue equal to 1 . Thus, price dynamics are characterized by interaction between unit root behavior far from the the fundamental (when EMH-believers dominate), with relatively small price changes driven only by exogenous news, and larger price changes due to amplification by trend following rules in some neighborhood of the fundamental price. ${ }^{2}$

\footnotetext{
${ }^{2}$ The deterministic skeletons of these examples have a stable steady state and a coexisting limit cycle. The dynamics along the cycle is characterized as follows. Let us start with a price slightly below $p^{*}$. Prices slowly increase and trend followers perform slightly better than EMH-believers. Thus their fraction increases, which reinforces the increase of prices and in turn the increase of the fraction of trend followers. During this phase returns increase and volatility jumps to a high value. However as prices deviate too much from the fundamental value the fraction of trend
} 

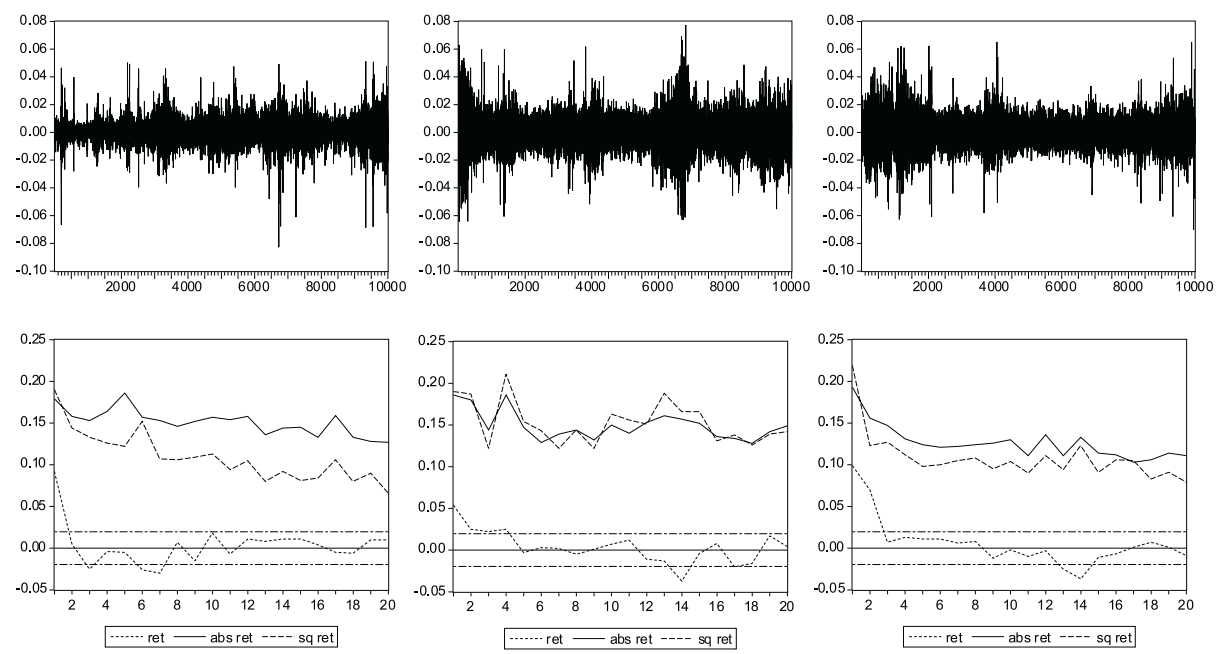

Fig. 2. Returns series (top panel) and autocorrelations of returns, absolute returns and squared returns (bottom panel) for daily S\&P 500 data $(08 / 17 / 1961-05 / 10 / 2000$, the October 1987 crash and the two days thereafter have been excluded; left panel) and data generated by our model. Middle: performance measure minus squared prediction errors (11), right: performance measure accumulated profits (10); parameter values: $\beta=2, R=1.001, v=1, g=1.89$ resp. $1.9, \bar{y}=1, p^{*}=1000, \alpha=2000$ resp. 1800 , $\eta=0$ resp. $0.99, a \sigma^{2}=1, \varepsilon_{t} \sim N(0,10), \delta_{t} \equiv 0$.

The return series of both simulations exhibit fat tails with kurtosis coefficient 5.39 resp. 5.58 (versus 8.51 for the S\&P 500). Autocorrelations for our simulations are very similar to those of the S\&P 500, with almost no significant autocorrelations in returns, but highly significant positive, slowly decaying autocorrelations in absolute and squared returns. That is, our model exhibits long memory and captures the phenomenon of volatility clustering (see [GH] for further details). ${ }^{3}$ Our examples also demonstrate that the characteristics of the model is very robust w.r.t the choice of the performance measure in (9).

\section{Conclusions}

We have modeled financial markets as nonlinear adaptive belief systems. Even in the simple case with only two types of traders, fundamentalists and technical traders, evolutionary competition between different trading strategies may

followers declines and EMH-believers dominate the market. This causes prices to decline and to move slowly in the direction of the fundamental price again, etc. (see $[\mathrm{GH}]$ ). Adding a dynamic noise term destroys the regularity, but clustered volatility remains.

${ }^{3}$ We get the strongest form of volatility clustering for $v=1$. However, our results are fairly robust w.r.t. other model parameters. [G2] presents examples for $v<1$ and with positive information costs for the fundamental predictor. 
generate endogenous fluctuations causing excess volatility. Our model is able to generate some of the stylized facts observed in many financial time series, such as unpredictability of returns, fat tails, and volatility clustering. These phenomena are created or reinforced by the trading process itself, which seems to be in line with common financial practice. Deviations from fundamentals triggered by small shocks may be reinforced by boundedly rational beliefs or strategies. Random news plays a role as a trigger event for volatility clustering, which may be amplified by evolutionary forces. This demonstrates that small fundamental causes may occasionally have big consequences and trigger large changes in asset prices. This observation is important for risk management and regulatory policies, but much more research in this area is needed for a better understanding of these forces.

Acknowlegdements. This research was supported by the Austrian Science Foundation (FWF) under grant SFB\#010 ('Adaptive Information Systems and Modelling in Economics and Management Science') and by the Netherlands Organization for Scientific Research (NWO) under a NWO-MaG Pionier grant.

\section{References}

[BH1] Brock, W.A., Hommes, C.H.: A rational route to randomness. Econometrica 65 (1997a) 1059-1095

[BH2] Brock, W.A., Hommes, C.H.: Models of complexity in economics and finance. In: Hey, C., Schumacher, J.M., Hanzon, B., Praagman, C. (eds.): System Dynamics in Economic and Financial Models. Wiley Publ., New York (1997b) $3-41$

[BH3] Brock, W.A., Hommes, C.H.: Heterogeneous beliefs and routes to chaos in a simple asset pricing model. Journal of Economic Dynamics and Control 22 (1998) 1235-74

[BH4] Brock, W.A., Hommes, C.H.: Rational Animal Spirits. In: Herings, P.J.J., Laan, van der G., Talman, A.J.J. (eds.): The Theory of Markets. NorthHolland, Amsterdam (1999) 109-137

[G1] Gaunersdorfer, A.: Endogenous fluctuations in a simple asset pricing model with heterogeneous beliefs. Journal of Economic Dynamics and Control 24 (2000) 799-831

[G2] Gaunersdorfer, A.: Adaptive Beliefs and the Volatility of Asset Prices. Central European Journal of Operations Research (2001) (to appear)

[GH] Gaunersdorfer A., Hommes C.H.: A Nonlinear Structural Model for Volatility Clustering. SFB Working Paper No. 63, University of Vienna and Vienna University of Economics and Business Administration, and CeNDEF Working Paper WP 00-02 (2000)

[GHW] Gaunersdorfer A., Hommes C.H., Wagener F.O.J.: Bifurcation routes to volatility clustering. SFB Working Paper No. 73, University of Vienna and Vienna University of Economics and Business Administration, and CeNDEF Working Paper WP 00-04 (2000)

[H] Hommes C.H.: Financial Markets as Nonlinear Adaptive Evolutionary Systems. Quantitative Finance 1 (2001) 149-167 